\title{
Estrés y desempeño ocupacional en estudiantes de terapia ocupacional.
}

\section{Stress and occupational performance in occupational therapy students}

\author{
Blanco Gisela $^{1,}$ Angulo Yamileth ${ }^{2}$, Contreras Jeilynn $^{3}$, Pacheco Yusana ${ }^{4}$, \\ Vargas Vanessa
}

\begin{abstract}
RESUMEN
Se trata de una investigación en la cual se estudió el Estrés Académico y el Desempeño Ocupacional en los estudiantes de la carrera de Terapia Ocupacional, de Universidad Central de Venezuela. El Objetivo fue determinar la relación existente entre el Estrés Académico y el Desempeño Ocupacional en los estudiantes de Terapia Ocupacional. Se combinaron técnicas de la metodología cuantitativa y cualitativa; para evaluar el estrés académico se utilizó la "Escala de Situaciones de Estrés Académico" (De Pablo, 2002 y adaptado por Feldman y col. 2008) y se diseñó una entrevista a profundidad basada en la Medida Canadiense de "Rendimiento Ocupacional". Participaron 101 estudiantes (73 mujeres y 28 hombres). Los estudiantes reportaron niveles moderados de estrés académico $(X=77,63)$ siendo la dimensión con mayor reporte el Estrés en los Exámenes $(X=30,91)$, seguido por el Estrés General $(X=20,97)$. Aquellos estudiantes que reflejaron niveles altos de estrés, en la entrevista reportaron que su ejecución en algunas áreas del Desempeño Ocupacional como el autocuidado, accesibilidad, escolaridad y socialización, resultaron ser insatisfactoria debido a la inadecuada alimentación, arreglo personal, actividades recreativas restringidas, todo ello por falta de tiempo, difícil acceso a la sede de la universidad y medios de transporte deficientes. Estos resultados permiten señalar que podría existir una relación entre Estrés Académico y el Desempeño Ocupacional del estudiante, ya que afectan de manera no satisfactoria la ejecución de las áreas de desempeño, roles y su participación activa en los diferentes contextos, rompiendo así el equilibrio vital.
\end{abstract}

Palabras claves: Estrés Académico, Desempeño Ocupacional, estudiantes.

\begin{abstract}
This in an investigation in wich was studied the Academic Stress and the Occupational Performance in students of the career Occupational Theraphy, Central University of Venezuela. The Objective was to determine the existing relation between Academic Stress and the Occupational Performance in the students of Occupational Therapy. Techniques of the quantitative and qualitative methodology were combined; in order to evaluate academic stress it was used the "scale of Situations of Stress Academic" (Of Pablo, 2002 and adapted by Feldman and col. 2008) and an interview to depth based on the Canadian Measurement of Occupational Yield was designed". 101 students participated ( 73 women and $28 \mathrm{men}$ ). The students reported moderate levels of academic stress $(X=77.63)$ being the dimension with greater report Stress in the Examinations ( $X=30.91)$, followed by General Stress $(X=20.97)$. Those students who reflected high levels of stress, in the interview reported that their execution in some areas of the Occupational Performance like the selfcare, accessibility, schooling and socialization, turned out to be unsatisfactory; due to the inadequate feeding, personal adjustment, restricted recreational activities, all it by lack of time, difficult access to the university location and poor transportation. These results allow to indicate that a relation between Academic Stress could exist and the Occupational Performance of the student, since they affect of nonsatisfactory way the execution of the performance areas, rolls and their active participation in the different contexts, breaking therefore the vital balance.
\end{abstract}

Key words: Academic stress, Occupational Performance, students.

\footnotetext{
${ }^{1}$ Profesora Asociada de la Escuela de Salud Pública, Universidad Central de Venezuela; Complejo Hospitalario José Ignacio Baldó El Algodonal, Antimano Caracas Venezuela. giblanco5@yahoo.com

${ }^{2}$ Terapeuta Ocupacional, Universidad Central de Venezuela

${ }^{3}$ Terapeuta Ocupacional, Universidad Central de Venezuela

${ }^{4}$ Terapeuta Ocupacional, Universidad Central de Venezuela

${ }^{5}$ Terapeuta Ocupacional, Universidad Central de Venezuela
} 


\section{INTRODUCCION}

La vida actual nos somete constantemente a presiones, que sin darnos cuenta nos provocan diversos grados de estrés, el cual se manifiesta generalmente como resultado de las frustraciones originadas por necesidades insatisfechas o también como respuestas a diversas situaciones. Para el organismo, el estrés tiene un significado bastante amplio, es un sinónimo de cambio, cualquier acontecimiento que cause un cambio en la vida sin importar que sea bueno o malo, real o imaginario, puede causar estrés.

Lazarus y Folkman (1984), consideraron el estrés como la evaluación cognitiva que hace la persona sobre un estímulo estresor, lo cual define la reacción o respuesta estresante. En el estrés están presentes procesos biológicos como la tensión y relajación, utilizados para el desempeño diario, entre ellos debe existir un equilibrio.

Se considera una importante fuente de estrés para los estudiantes universitarios, el conjunto de actividades académicas propias de la universidad que pueden influir sobre su bienestar físico, psicológico, el despliegue de conductas saludables e igualmente causa un efecto sobre el rendimiento (Fedlman, Goncalves, Chacón, Zaragoza, Bagés, De Pablo; 2008). En base a los estudios de Orlandini (1999), conceptualiza el estrés académico, como aquel que padecen los estudiantes a consecuencia de las exigencias endógenas o exógenas que impactan su desempeño en el ámbito universitario.

Otra definición, explica el estrés académico como un proceso sistémico, de carácter adaptativo y esencialmente psicológico, que se presenta: a) cuando el estudiante se ve sometido, en contextos escolares, a una serie de demandas que, bajo la valoración del propio estudiante son considerados estresores (input); b) cuando estos estresores provocan un desequilibrio sistémico (situación estresante) que se manifiesta en una serie de síntomas (indicadores del desequilibrio); y c) cuando este desequilibrio obliga al estudiante a realizar acciones de afrontamiento (output) para restaurar el equilibrio sistémico. (Orlandini, 1999)

Los estudiantes se enfrentan cada vez más a exigencias, desafíos y retos que les demandan una gran cantidad de recursos físicos y psicológicos de diferente índole. Tal situación le puede hacer experimentar agotamiento, poco interés frente al estudio, autocrítica e incluso, llevarlo a la pérdida de control sobre el ambiente, aunque sea sólo de manera transitoria. (Lazarus y Folkman, 1984)

Con la idea de avanzar en una conceptualización multidimensional del estrés académico, Orlandini (1999) creó un modelo que toma como base la teoría general de sistemas y la teoría transaccional del estrés, llamado modelo sistémico cognoscitivista para el estudio del estrés académico, el cual permite caracterizar al estrés académico como un estado psicológico de la persona.

El modelo sistémico cognoscitivo para el estudio del estrés académico se basa, en que el entorno le plantea al estudiante un conjunto de demandas o exigencias, estas demandas son sometidas a un proceso de valoración por parte de la persona, en el caso de considerar que las demandas desbordan sus recursos las valora como estresores, los cuales constituyen el 
input que entra al sistema y provoca un desequilibrio sistémico en la relación del estudiante con su entorno, el desequilibrio sistémico da paso a un segundo proceso de valoración de la capacidad de afrontar la situación estresante, lo que determina cuál es la mejor manera de enfrentar esa situación, el sistema responde con estrategias de afrontamiento (output) a las demandas del entorno y una vez aplicadas estas estrategias de afrontamiento, en caso de ser exitosas, el sistema recupera su equilibrio sistémico; en caso de que las estrategias de afrontamiento no sean exitosas, el sistema realiza un tercer proceso de valoración que lo conduce a un ajuste de las estrategias para lograr el éxito esperado. (Orlandini, 1999)

Se han llevado a cabo diversas investigaciones sobre el estrés académico entre ellas está la de Barraza (2006); el cual refiere que el estrés por un examen se ha convertido en objeto de investigación y aborda la conceptualización del tema desde el modelo sistémico cognoscitivo. Tanto para los profesores, como entre los estudiantes, se considera como algo normal que el estudiante esté algo nervioso, estresado o ansioso antes de iniciar un examen; esta situación puede ayudar a que el alumno esté más alerta y despliegue mayor esfuerzo de concentración y acción, lo cual le ayudaría a mantener un rendimiento alto mientras hace su prueba. Sin embargo, en algunos casos el estrés es muy intenso, a tal grado que interfiere en la concentración y el rendimiento.

Este mismo autor (Barraza, 2006), realizó una de las primeras investigaciones que abordaron el estrés que se genera a partir de un examen. Estudió la experiencia de un grupo de estudiantes en torno a la preparación y realización de sus exámenes de doctorado. Bajo esta misma lógica se han realizado múltiples investigaciones que abordan el estrés a partir de una situación generada por un examen. La lógica subyacente de estas investigaciones, es que parten del hecho de reconocer el examen como una situación generadora de estrés; sin embargo, ninguna ha estudiado al examen como una situación estresante en sí misma. Esta circunstancia conduce a abordar el estudio del estrés de examen y a considerarlo un tipo de estrés derivado del estrés académico.

Con base en el modelo sistémico cognoscitivo del estrés académico, es posible conceptualizar el estrés de examen como un proceso sistémico, de carácter adaptativo y esencialmente psicológico. Esta conceptualización implica aceptar de manera explícita las hipótesis constituyentes de este modelo: hipótesis de los componentes sistémicosprocesuales del estrés académico, hipótesis del estrés académico como estado psicológico, hipótesis de los indicadores del desequilibrio sistémico que implica el estrés académico y la hipótesis del afrontamiento como restaurador del equilibrio sistémico. (Barraza 2007)

Según estudios realizados en las carreras pertenecientes a las ciencias de la salud, se ha evidenciado la presencia de altos niveles de estrés. Esto se muestra a partir de las estadísticas reportadas por el continente Africano donde ubican la incidencia de estrés en un $64,5 \%$ en estudiantes de primer año de Medicina. Así mismo, en el continente Americano son diversas las investigaciones publicadas al respecto, destacándose países como Chile, México, Perú y Venezuela, donde se demuestra una elevada incidencia de estrés en estudiantes universitarios, alcanzando valores superiores al $67 \%$ de la población estudiada en la categoría de estrés moderado. (Román, Ortiz, Hernández, 2008) 
Una investigación sobre estrés, realizada en dos grupos de participantes: odontólogos y estudiantes del último año de la carrera de odontología, arrojó como resultado, que los estudiantes del último año de la carrera, presentan puntuaciones más elevadas en los factores cansancio emocional y despersonalización. En los odontólogos se detectó mayor realización personal en su trabajo (Albanesi, Tifner, Nasetta, 2006).

Rajab (2001), encontró que en estudiantes de odontología, los mayores productores de estrés son los exámenes, los días cargados de trabajo y la falta de tiempo para el descanso. En los años clínicos, el bienestar de los pacientes o el no cumplimiento de sus turnos, junto con el cumplimiento de requisitos académicos fueron los principales factores. Existen según este autor diferencias entre los años clínicos y teóricos, considera que otros factores que influyen son: el medio ambiente educacional, aspectos culturales y/o personales.

Entre otros estudios, se encuentra el realizado sobre la evaluación de situaciones generadoras y manifestaciones de estrés académico en estudiantes de tercero y cuarto año de una Facultad de Estomatología. Los resultados revelaron que el 96,6\% de estudiantes reconocieron la presencia de estrés académico, siendo los de tercer año los que presentaron los niveles más altos, (las mujeres presentaron niveles más bajos de estrés en relación a los varones). Las situaciones mayormente generadoras de estrés fueron tiempo para cumplir con las actividades, sobrecarga de tareas, trabajos de cursos y responsabilidad por cumplir obligaciones académicas. Las manifestaciones físicas, psicológicas y comportamentales se presentaron con mayor intensidad en los varones; siendo las psicológicas, las que tuvieron mayor presencia e intensidad en ambos sexos y años de estudios. Finalmente se encontró que el nivel de estrés académico se relacionó positivamente con todas las situaciones generadoras y manifestaciones físicas y psicológicas. (Bedoya, Perea, Ormeño, 2006)

Sajjan y Krupa (2005), encontraron que un grupo de estudiantes de postgrado de Terapia Ocupacional, refirió que el estrés estuvo asociado a la incertidumbre de sus perspectivas futuras que incluyen las oportunidades de empleo cuando terminen sus programas académicos. Señalaron que los estudiantes de postgrado tienden a carecer del tiempo y/o de la oportunidad de desarrollar las relaciones interpersonales. Por otra parte hicieron hincapié que otras tareas que producen estrés son los exámenes, proyectos de trabajo, tesis y el miedo al fracaso académico.

No hay duda que el estrés es un efecto psicosocial de gran impacto en la actualidad, especialmente por su relación con la aparición de cierto tipo de enfermedades, el manejo y adaptación a situaciones difíciles; genera a su vez alteraciones significativas en el desempeño ocupacional, lo que significa que puede existir una ruptura o desequilibrio en los diferentes contextos ocupacionales. Los seres humanos son seres ocupacionales por naturaleza; sin embargo la ocupación humana es paradójica, al ser un fenómeno universal y único, la misma ocupación no tiene igual significado ni es experimentada de la misma forma por dos personas. (Simó y Urbanowski, 2006)

Para Law, Polatajko, Baptiste (1997) la relación entre los sujetos, el entorno y la ocupación cambian a lo largo de la vida en respuesta a las oportunidades y desafíos que dan forma al transcurso de la vida ocupacional de cada persona. Más específicamente, el desempeño ocupacional se concibe como el resultado de una relación dinámica entre personas, entorno 
y ocupación a lo largo de la vida del sujeto y cualquier cambio en cualquier parte de la interacción entre sujeto, entorno y ocupación afecta a las otras partes y en consecuencia, al desempeño (Kielhofner, 2006). También se refiere a la capacidad de la persona de elegir, organizar y desarrollar de forma satisfactoria ocupaciones significativas y culturalmente adaptadas, en búsqueda del cuidado personal, del disfrute de la vida y contribución a la sociedad, es un proceso dinámico reflejado en la constante interacción de los elementos que lo conforman.

Para detectar dificultades en el desempeño ocupacional se cuenta con uno de los modelos propios de la Terapia Ocupacional como es, el Modelo Canadiense de Desempeño Ocupacional que fue desarrollo durante la década de los 80, por terapeutas ocupacionales canadienses con la finalidad de llevar a la práctica la filosofía de una terapia centrada en el cliente como un primer enfoque y como segundo enfoque el desempeño ocupacional (Simó y Urbanowski, 2006). Se basa en una perspectiva centrada en la persona, recobrando así las raíces humanistas de la profesión. Al hablar del cliente se refiere tanto a personas a escala individual, como a grupos, empresas o comunidades.

El concepto de ocupación se define según el modelo, como el término general de ocuparse de sí mismo y de ejercer el control en la propia vida. La ocupación es una necesidad humana básica. Toda persona debe estar ocupada en las distintas facetas de su vida. La salud como concepto extendido permite tener las oportunidades, las habilidades para involucrarse en patrones relevantes de ocupación para el cuidado de si mismo, así como la participación social y económica a lo largo de la vida; más allá de la ausencia de enfermedad. (Kielhofner, 2006)

Durante el proceso de desarrollo y desempeño ocupacional, se generan transformaciones positivas y negativas, desencadenando cambios personales, ocupacionales y/o del entorno. Estos cambios inciden de forma directa, provocando un desequilibrio en la salud socioemocional, en el temperamento, en la personalidad, energía, impulsos y funciones emocionales; todo ello a consecuencia del estrés. (Crepeau, Cohn, Boyt, 2006)

Los aspectos antes desarrollados permiten inferir que el estrés académico podría incidir directamente en el desempeño ocupacional, específicamente en el área de productividad, según el modelo canadiense, el cual se refiere a la capacidad para ejecutar aquellas tareas que hacen posible desarrollar los roles ocupacionales de una manera que sea satisfactoria y apropiada en relación con la edad del individuo, la cultura y el entorno (Polonio, 2001).

\section{METODO}

Se trató de una investigación donde se combinó la metodología cualitativa y cuantitativa. Cualitativa, ya que se estudió la asociación en contextos estructurales y situacionales, se 
trató de identificar la naturaleza profunda de algunos fenómenos tal y como son experimentados, vividos y percibidos por el ser humano, además de las realidades, su sistema de relaciones y su estructura dinámica, es decir desde una perspectiva fenomenológica (Martínez, 1996). En esta investigación se trató de indagar el significado, las consecuencias y cómo experimentan, este grupo de estudiantes, el impacto del estrés académico en su desempeño ocupacional.

Se consideró cuantitativa de tipo descriptiva, ya que consistió en la caracterización de un hecho; en este caso el estrés académico. Con un diseño no experimental, porque sólo se limitó a la observación sin la manipulación deliberada de la variable estrés académico.

La población total fue de 101 estudiantes, con una edad promedio de 21,54 años (ver Tabla 1), cursantes de los 4 años de la Licenciatura de Terapia Ocupacional de la Universidad Central de Venezuela, correspondiente al período lectivo 2010-2011, en cuanto al sexo, 73 fueron mujeres y 28 hombres.

TABLA N $\mathbf{N}^{\circ} 1$

Promedio de edad de toda la población y por años de la carrera

\begin{tabular}{lcccc}
\hline & Media & $\begin{array}{c}\text { Desviación } \\
\text { Típica }\end{array}$ & Mínimo & Máximo \\
\hline Toda la población & 21,54 & 4,929 & 16 & 42 \\
$1^{\circ}$ Año & 19,76 & 3,748 & 16 & 31 \\
$2^{\circ}$ Año & 20,11 & 2,374 & 18 & 28 \\
$3^{\circ}$ Año & 21,67 & 3,841 & 19 & 31 \\
$4^{\circ}$ Año & 24,03 & 6,657 & 20 & 42 \\
\hline
\end{tabular}

$\mathbf{N}=\mathbf{1 0 1}$

Posteriormente se seleccionó una muestra no probabilística de tipo intencional, cuyo criterio de inclusión implicó seleccionar sólo aquellos estudiantes de Terapia Ocupacional que presentaran estrés académico, los cuales participaron en la segunda fase de la investigación donde se les aplicó una entrevista semi-estructurada, para estudiar las relaciones entre el estrés académico y el desempeño ocupacional. Siendo un total de 16 estudiantes cursantes de los 4 años de la carrera, en donde 15 fueron mujeres, 1 hombre: En cuanto a la distribución por año se obtuvo 4 pertenecientes al primer año, 3 al segundo año, 4 al tercer año y 5 al cuarto año.

Las variables que se estudiaron fueron el estrés académico: considerado como la activación de una serie de respuestas fisiológicas y conductuales en el organismo del estudiante universitario ante las demandas académicas (Pfeifer, Kranz, Scoggin, 2008). Y el desempeño ocupacional según el Modelo Canadiense de Desempeño Ocupacional, el cual 
se refiere a la capacidad de elegir, organizar y desempeñar de manera satisfactoria ocupaciones significativas definidas por la cultura y adecuadas a la edad para el autocuidado, el disfrute de la vida y para contribuir con el entramado social y económico de la comunidad. (Polonio, 2001)

Para la evaluación del estrés académico se utilizó la "Escala de Situaciones de Estrés Académico del Hospital Clínico-Barcelona-España”, fue elaborada por De Pablo, en el año 2002 y adaptado por Feldman et al.( 2008). Consta de 18 ítems que evalúan la intensidad con que el individuo percibe las fuentes de estrés más importantes de la vida académica. Cada posible situación se puntúa a través de una escala de Likert de 10 puntos $(0=$ Nada estresante y $9=$ Muy estresante). Además, se recoge si la situación estresante se ha producido en las cuatro últimas semanas. De este modo se pueden obtener dos puntuaciones básicas a través de la calificación del cuestionario. El cuestionario demostró tener niveles apropiados de confiabilidad, tanto en muestras españolas como venezolanas (Alpha de Cronbach oscila entre 0.78 y 0.90) (Albanesi et al. 2006). Cabe destacar que para esta investigación sólo se consideró el Estrés Global y sus dimensiones, obteniéndose un valor de fiabilidad de 0,85 .

Por otra parte para conocer el desempeño ocupacional del grupo estudiado se utilizó "La Medida Canadiense de Desempeño Ocupacional" (COPM), elaborada por Law et al., en el año 1997. Es una medida personalizada, diseñada para ser usada por terapeutas ocupacionales en la identificación de cambios en la auto-percepción del cliente de su rendimiento ocupacional a lo largo del tiempo. La COPM tiene el objetivo de ser utilizada como una medida de resultados y como tal debe administrarse al inicio de la intervención en Terapia Ocupacional y repetirse periódicamente a intervalos determinados por el cliente y el terapeuta. Para la presente investigación se realizó una adaptación de la Medida antes descrita, elaborada en el año 2010 por las autoras de este trabajo. La adaptación consiste en una entrevista semi-estructurada, en donde se exploró en el estudiante, información acerca de sus actividades de autocuidado, escolaridad y ocio. Se le solicitó que identifique cuál(es) ejecuta y vivencia de forma satisfactoria, cuál(es) no y por qué.

\section{RESULTADOS}

En la Tabla 2, se presentan los resultados del estrés global y de los componentes del estrés académico del total de participantes, en donde se pudo observar que el grupo de estudiantes participantes reportan niveles moderados de estrés académico $(X=77,63)$ y el componente con mayor reporte de estrés por los estudiantes fue Estrés en los Exámenes ( $X=30,91)$, seguido por el componente Estrés General $(X=20,97)$ y en el componente Ansiedad Social $(X=20,89)$ se observaron bajos niveles de estrés.

TABLA No 2

Estrés académico global y componentes del estrés académico del total de participantes 


\begin{tabular}{lcccc}
\hline & Media & $\begin{array}{c}\text { Desviación } \\
\text { Típica }\end{array}$ & Mínimo & Máximo \\
\hline Estrés Global & 77,63 & 25,394 & 15 & 139 \\
Estrés General & 20,97 & 10,204 & 4 & 47 \\
Ansiedad Social & 20,89 & 9,450 & 0 & 42 \\
Estrés en los Exámenes & 30,91 & 9,644 & 0 & 45 \\
\hline
\end{tabular}

$\mathbf{N}=\mathbf{1 0 1}$

En relación a los resultados del estrés global y los componentes del estrés académico de los estudiantes que cursan el primer año de la carrera, se reportan niveles moderados de estrés académico $(X=62,72)$ y el componente con mayor reporte fue Estrés en los Exámenes $(X=$ 27,14), seguido por el componente de Ansiedad Social $(X=17,28)$ y por último el Estrés General $(\mathrm{X}=12,76)$. (Ver Tabla 3)

TABLA N 3

Estrés académico global y componentes del estrés académico del $1^{\circ}$ Año de la Carrera de Terapia Ocupacional

\begin{tabular}{lcccc}
\hline & Media & $\begin{array}{c}\text { Desviación } \\
\text { Típica }\end{array}$ & Mínimo & Máximo \\
\hline Estrés Global & 62,72 & 22,223 & 15 & 103 \\
Estrés General & 12,76 & 6,523 & 4 & 27 \\
Ansiedad Social & 17,28 & 8,757 & 3 & 37 \\
Estrés en los Exámenes & 27,14 & 9,862 & 0 & 42 \\
\hline
\end{tabular}

$\mathbf{N}=\mathbf{2 9}$

En la Tabla 4, se observan los resultados del estrés global y de los componentes del estrés académico de los estudiantes que cursan el segundo año de la carrera, en donde se muestra que la población se encuentra con niveles altos de estrés académico $(X=80,33)$ y el componente con mayores niveles reportados fue Estrés en los Exámenes (X=33,33), seguido por el componente Estrés General $(X=24,61)$ y por último la Ansiedad Social $(X=$ 18,44).

TABLA $\mathbf{N}^{\circ} 4$

Estrés académico global y componentes del estrés académico del $2^{\circ}$ Año de la Carrera de Terapia Ocupacional 


\begin{tabular}{lcccc}
\hline & Media & $\begin{array}{c}\text { Desviación } \\
\text { Típica }\end{array}$ & Mínimo & Máximo \\
\hline Estrés Global & 80,33 & 19,656 & 52 & 121 \\
Estrés General & 24,61 & 8,325 & 12 & 44 \\
Ansiedad Social & 18,44 & 9,231 & 0 & 35 \\
Estrés en los Exámenes & 33,33 & 9,312 & 15 & 45 \\
\hline
\end{tabular}

$\mathbf{N}=18$

En cuanto a los resultados del estrés global y los componentes del estrés académico de los estudiantes que cursan el tercer año de la carrera, en donde se muestra que la población reportó niveles moderados de estrés académico $(\mathrm{X}=77,08)$ y el componente con mayor reporte fue Estrés en los Exámenes $(X=29,13)$, seguido por el componente Estrés General $(X=23,04)$ y por último la Ansiedad Social $(X=20,79)$. (Ver Tabla 5)

TABLA N $\mathbf{N}^{\circ}$

Estrés académico global y componentes del estrés académico del $3^{\circ}$ Año de la Carrera de Terapia Ocupacional

\begin{tabular}{lcccc}
\hline & Media & $\begin{array}{c}\text { Desviación } \\
\text { Típica }\end{array}$ & Mínimo & Máximo \\
\hline Estrés Global & 77,08 & 26,475 & 34 & 133 \\
Estrés General & 23,04 & 9,224 & 10 & 46 \\
Ansiedad Social & 20,79 & 9,628 & 3 & 39 \\
Estrés en los Exámenes & 29,13 & 10,535 & 7 & 44 \\
\hline
\end{tabular}

$\mathrm{N}=\mathbf{2 4}$

En relación a los resultados de los estudiantes que cursan cuarto año se puede observar en la Tabla 6, que los participantes reportaron altos niveles de estrés académico $(X=90,87)$, resultando que el componente con mayor reporte fue Estrés en los Exámenes $(X=34,53)$, seguido por el componente Ansiedad Social $(X=25,73)$ y por último la de Estrés General $(X=25,07)$.

TABLA N ${ }^{\circ} 6$

Estrés académico global y componentes del estrés académico del $4^{\circ}$ Año de Terapia Ocupacional 


\begin{tabular}{lcccc}
\hline & Media & $\begin{array}{c}\text { Desviación } \\
\text { Típica }\end{array}$ & Mínimo & Máximo \\
\hline Estrés Global & 90,87 & 23,585 & 52 & 139 \\
Estrés General & 25,07 & 10,645 & 9 & 47 \\
Ansiedad Social & 25,73 & 8,416 & 10 & 42 \\
Estrés en los Exámenes & 34,53 & 7,338 & 16 & 45 \\
\hline
\end{tabular}

$\mathbf{N}=\mathbf{3 0}$

En cuanto a los resultados de la entrevista realizada mediante la adecuación de la Medida Canadiense de Desempeño Ocupacional, se muestran a través de un cuadro que contiene las dimensiones, descripción y aquellos aspectos que con más frecuencia reportaron los 16 estudiantes seleccionados, a partir de las puntuaciones máximas alcanzadas en la Escala de Situaciones de Estrés Académico.

\section{CUADRO No 1}

Resultados de la Adecuación de la Medida Canadiense de Desempeño Ocupacional

\begin{tabular}{|c|c|c|}
\hline Dimensión & Descripción & Lo reportado \\
\hline Autocuidado & $\begin{array}{l}\text { Se refiere al cuidado } \\
\text { personal, vestido, baño, } \\
\text { alimentación e higiene. }\end{array}$ & $\begin{array}{l}\text {-Inadecuada alimentación y } \\
\text { poco arreglo personal, debido a } \\
\text { falta de tiempo. } \\
\text {-Comer a deshoras. }\end{array}$ \\
\hline Accesibilidad & $\begin{array}{l}\text { Tiempo de traslado de la } \\
\text { casa a la universidad y } \\
\text { viceversa, al igual que } \\
\text { toma en consideración el } \\
\text { medio de transporte. }\end{array}$ & $\begin{array}{l}\text { - Retrasos en el metro. } \\
\text {-Las colas del tráfico. } \\
\text {-Los diferentes medios de } \\
\text { transporte que deben utilizar. } \\
\text { - Dificultad en el acceso a la } \\
\text { Escuela de Salud Pública, } \\
\text { debido a su ubicación. }\end{array}$ \\
\hline $\begin{array}{l}\text { Manejo de la } \\
\text { Comunidad }\end{array}$ & $\begin{array}{lr}\text { Participación } & \text { en } \\
\text { actividades } & \text { como; } \\
\text { compras, } & \text { asuntos } \\
\text { familiares, de } & \text { la } \\
\text { comunidad y financieros. }\end{array}$ & $\begin{array}{l}\text {-Antes de su ingreso a la } \\
\text { universidad les era posible } \\
\text { participar en este tipo de } \\
\text { actividades, ahora cuentan con } \\
\text { un tiempo restringido, el cual lo } \\
\text { emplean para estudiar y/o hacer } \\
\text { trabajos académicos. }\end{array}$ \\
\hline
\end{tabular}




\begin{tabular}{|c|c|c|}
\hline Escolaridad & $\begin{array}{l}\text { Relacionado con la } \\
\text { puntuación promedia del } \\
\text { estudiante, asignación de } \\
\text { trabajos escritos, número } \\
\text { de materias vistas por año, } \\
\text { la asignación de un } \\
\text { calendario de exámenes y } \\
\text { la carga horaria. }\end{array}$ & $\begin{array}{l}\text {-Estudiantes acostumbrados a } \\
\text { obtener un promedio alto en el } \\
\text { bachillerato. } \\
\text {-Genera tensión entre los } \\
\text { estudiantes ver la cantidad de } \\
\text { exámenes programados y no } \\
\text { tener tiempo suficiente para } \\
\text { estudiar. }\end{array}$ \\
\hline $\begin{array}{c}\text { Manejo de las } \\
\text { Actividades de la Vida } \\
\text { Diaria }\end{array}$ & $\begin{array}{l}\text { Participación en las } \\
\text { actividades relacionadas al } \\
\text { hogar como; limpieza, } \\
\text { arreglo y preparación de } \\
\text { los alimentos. }\end{array}$ & $\begin{array}{l}\text {-Desde que comenzaron la } \\
\text { universidad se olvidaron de } \\
\text { realizarlas y si las realizan lo } \\
\text { hacen esporádicamente. }\end{array}$ \\
\hline $\begin{array}{c}\text { Actividades Recreativas } \\
\text { Estáticas }\end{array}$ & $\begin{array}{l}\text { Se refiere a la } \\
\text { participación } \\
\text { actividades como; lectura, } \\
\text { navegación en la red y } \\
\text { conectarse a redes } \\
\text { sociales. }\end{array}$ & $\begin{array}{l}\text {-No las realizan por falta de } \\
\text { tiempo, debido a las actividades } \\
\text { de la universidad. } \\
\text {-La red de internet es utilizada } \\
\text { para investigaciones y no como } \\
\text { medio de recreación. }\end{array}$ \\
\hline $\begin{array}{c}\text { Actividades Recreativas } \\
\text { Activas }\end{array}$ & $\begin{array}{l}\text { Participación del } \\
\text { estudiante en actividades } \\
\text { como; deportes, } \\
\text { excursiones y viajes. }\end{array}$ & $\begin{array}{l}\text {-Este tipo de actividades quedan } \\
\text { a un lado, por las actividades } \\
\text { requeridas por la universidad. }\end{array}$ \\
\hline Socialización & $\begin{array}{l}\text { Incluye la participación en } \\
\text { actividades como; hacer } \\
\text { visitas, llamar por } \\
\text { teléfono, ir a fiestas, a la } \\
\text { playa y al cine. }\end{array}$ & $\begin{array}{l}\text {-Este tipo de actividades las } \\
\text { realizan esporádicamente } \\
\text { durante periodos de vacaciones. }\end{array}$ \\
\hline
\end{tabular}

Las siguientes dimensiones fueron reportadas como no satisfactorias por los estudiantes; presentándose a continuación algunos de los comentarios de las entrevistas:

\section{Auto-cuidado:}

"Debido a que siento que no tengo el suficiente tiempo para arreglarme, lo hago a medias". "No me da tiempo suficiente para cocinar"

"A veces debido a la carga horaria y a la cantidad de cosas por hacer no me da tiempo para ejecutarla".

"A veces me acuesto sin comer por el tiempo que requiere la universidad".

"No tengo la misma cantidad de tiempo que tenía antes para arreglarme, y no me da tiempo para cumplir con las tres comidas diarias, sólo almuerzo y meriendo".

"Sufro de gastritis, por el mal hábito de alimentación, es que no me da tiempo". 


\section{Accesibilidad:}

"Es estresante el traslado, más que todo los lunes".

"Utilizo el metro y siempre hay retraso".

"En la zona donde vivo se hace demasiada cola, son continuas y eternas, llevas golpes, gritos, qué no llevo...".

"Para llegar a la universidad necesito salir dos horas antes de la casa".

"Lo considero agotador y terrible, por las dificultades del metro, me retrasa las demás actividades".

"El número de transportes que debo tomar, son incómodas y generan mucho estrés".

\section{Manejo de la Comunidad:}

"El tiempo que utilizaba para eso, ahora lo invierto en cuestiones de la universidad".

"No tengo tiempo para realizar esas actividades, debido al tiempo que paso en la universidad".

"No me da tiempo debido a que tengo que estudiar o realizar trabajos para la universidad". "He tenido que dejar de participar en ellas por dedicarme a la universidad".

"Me falta tiempo porque tengo que hacer tareas y es lo más importante, las otras cosas pueden esperar".

\section{Escolaridad:}

"Me estreso muchísimo al ver la cantidad de exámenes y saber que no tengo tiempo suficiente para estudiar cómo se debe".

"A veces no me da tiempo de estudiar para todos los exámenes asignados".

"Si veo que tengo muchos exámenes seguidos eso me preocupa y me estresa, y paso las noches sin dormir pensando en eso".

"Llego a mi casa muy tarde".

"En comparación al primer año, tengo más bajo rendimiento y ahorita saco notas que no deseo".

"Obviamente hago un esfuerzo para tratar de obtener una buena nota y siempre es más bajo".

"Me produce mucha tensión, es una forma de presión".

"Me resulta estresante saber que tengo mucho que estudiar".

"Resulta estresante por la cantidad de materias y porque aunado a eso tenemos que culminar trabajos".

"Mucho tiempo de estudio genera agotamiento mental y se pierde la motivación, es difícil captar las ideas de las clases".

Manejo de las AIVD:

"Desde que entré en la universidad me olvide de todo eso".

"No me da tiempo de realizarlas varias veces al mes como me gustaría, porque tengo que llegar a la casa a estudiar".

"No me da tiempo de limpiar, llego muy cansada por el trayecto del lugar de pasantías a mi casa".

Actividades Recreativas Estáticas: 
"Tengo como un mes que no abro el messenger ni el facebook, sólo utilizo la red de internet para investigar".

"No me da tiempo para realizarlo".

"Utilizo el tiempo de las mismas es para realizar actividades académicas".

\section{Actividades Recreativas Activas:}

"No lo realizo debido a la universidad y por factores económicos".

"No participo en ir a trotar, en mis actividades de recreación no participo".

"Ni hablar pero tengo que agarrar un tiempo para salir, porque si no puedo explotar".

\section{Socialización:}

"Las puedo realizar sólo en vacaciones, de resto me resulta difícil".

"Pasar un día en la calle me quita tiempo para realizar las cosas de la universidad".

"No he ido a la playa, no voy a fiestas, los fines de semana llamo a mi mamá a Cumaná y eso me alegra la semana".

"De pronto puedo ir a la playa pero igual estoy con la angustia de que tengo que irme rápido y no duermo".

\section{DISCUSION}

Los resultados obtenidos en esta investigación muestran que los estudiantes que reflejaron niveles altos de estrés, luego en la entrevista reportaron que su ejecución en algunas áreas del Desempeño Ocupacional como el autocuidado, accesibilidad, escolaridad y socialización, resultaron ser insatisfactorias, debido a la inadecuada alimentación, poco arreglo personal, actividades recreativas restringidas, todo ello por falta de tiempo, además del difícil acceso a las instalaciones del núcleo universitario, medios de transportes deficientes, entre otros factores.

Son diversas las investigaciones que muestran una elevada incidencia de estrés en estudiantes universitarios, categorizándose como estrés moderado (Feldman, et al. 2008). En investigaciones realizadas en odontólogos y en estudiantes del último año de la carrera de odontología, arrojaron como resultados elevados niveles de estrés (Albanesi, et al. 2006)

Una investigación realizada en estudiantes de tercero y cuarto año de Estomatología, reveló que presentaron niveles altos de estrés, presentándose como situaciones generadoras del mismo el tiempo para cumplir con las actividades, sobrecarga de tareas y la asignación de trabajos de cursos, lo que influyó directamente en los factores físicos y psicológicos de la población objeto de estudio (Bedoya, et al. 2006).

En una investigación realizada en Terapeutas Ocupacionales de post-grado, se observó que los estudiantes reportaron niveles moderados de estrés, debido a factores como: abrumadoras cargas de trabajo, falta de claridad en las expectativas, preocupación por la distribución del tiempo y la transición de un nivel académico a otro, alterando de esta manera la ejecución de los roles (Pfeifer, Kranz y Scoggin, 2008) 
En la India se llevó a cabo un estudio con el propósito de cuantificar el estrés de pregrado y postgrado en los estudiantes de Terapia Ocupacional, obteniendo como resultados niveles altos de estrés, siendo mayor en los estudiantes de pregrado (Kielhofner, 2006).

En cuanto a la relación entre el estrés académico y el desempeño ocupacional de estudiantes, no se encontraron estudios que relacionen ambas variables. Sin embargo los estudios citados anteriormente señalan que los estudiantes (tanto de pre-grado como de post-grado) reportan niveles de estrés señalando las dimensiones o factores que influyen en su aparición.

Es así como tomando en consideración los antecedentes descritos y la investigación realizada, se puede determinar que existe una relación importante entre el estrés académico y el Desempeño Ocupacional, que incide de manera no satisfactoria en los estudiantes a nivel físico, psicológico y conductual, ya sea tratándose de pregrado o postgrado, sin importar ubicación geográfica, contexto cultural, ni sexo.

La investigación se llevó a cabo en dos fases. La primera consistió en la aplicación de la "Escala de Situaciones de Estrés Académico" a la población total de estudio, que fue de 101 estudiantes, de los cuales 16 de ellos resultaron con niveles altos de estrés, obteniendo así la muestra intencional para la realización de la segunda fase, en donde se utilizó la adecuación de la "Medida Canadiense de Desempeño Ocupacional", para determinar la incidencia del estrés académico en las áreas de desempeño del estudiante, reportando de esta manera que todas las dimensiones se encuentran afectas pero las de mayor incidencia y generadoras de estrés fueron: autocuidado, accesibilidad, escolaridad y socialización.

Con base en el análisis de los resultados arrojados por la presente investigación, se puede establecer que existe influencia del estrés académico y de los componentes que lo conforman sobre el desempeño ocupacional del estudiante, ya que afectan de manera no satisfactoria la ejecución de las áreas de desempeño, roles y su participación activa en los diferentes contextos, quebrantando así el equilibrio vital, entendiéndose éste, como el equilibrio entre los factores biopsicosociales que toda persona desea alcanzar a lo largo de su vida.

Los resultados también permiten sugerir que se estudie la posibilidad de modificar el pensum de estudio en cuanto a la distribución del número de asignaturas por año y carga horaria, así como modificar el modelo educativo tradicional de horas presenciales en el aula, en el cual el estudiante además de cumplir con la carga horaria establecida por el pensum, debe realizar una serie de actividades fuera de las horas de clase, afectando de esta manera el tiempo que puede ser utilizado para otras actividades. 


\section{REFERENCIAS BIBLIOGRAFICAS}

Albanesi S. Tifner S. Nasetta J. (2006). Estrés en odontología. Acta Odontológica Venezolana [Revista en Internet]. Diciembre; V. 44 (N3). Disponible en: http://www.scielo.org.ve/scielo.php?pid=S000163652006000300003\&script=sci arttext\&tl $\underline{\text { ng}}=\mathrm{es}$

Barraza MA. (2006). Un modelo conceptual para el estudio del estrés académico. Revista Electrónica Psicología Científica.com. Disponible en: http://www.psicologiacientifica.com/bv/psicologia-167-1-un-modelo-conceptual-para-elestudio-del-estres-academico.html

Barraza MA. El estrés de examen. (2007). Revista Electrónica Psicología Científica.com. Disponible en: http://www.psicologiacientifica.com/bv/psicologia-306-1-el-estres-deexamen.html

Bedoya LS. Perea PM. Ormeño MR. (2006). Evaluación de niveles, situaciones generadoras y manifestaciones de estrés académico en alumnos de tercer y cuarto año de una Facultad de Estomatología. Revista Estomatológica Herediana. Enero-Junio; V.16 $\left(\mathrm{N}^{\mathrm{o}} 1\right)$. Disponible en: http://revistas.concytec.gob.pe/scielo.php?script=sci_arttext\&pid=S1019435520060001000 $\underline{04 \& \operatorname{lng}=\text { es\&nrm=iso }}$

Crepeau EB. Cohn ES. Boyt BA. (2006) Terapia Ocupacional. 10 a ed. Buenos Aires: Médica Panamericana.

De Pablo J. Baillés E. Pérez J. Valdés M. (2002) Construcción de una escala de estrés académico para estudiantes universitarios. Enero; V.05 ( $\left.{ }^{\circ} 1\right)$ : [pp 40-46].

Feldman L. Goncalves L. Chacón G. Zaragoza J. Bagés N. De Pablo J. (2008). Relaciones entre estrés académico, apoyo social, salud mental y rendimiento académico en estudiantes universitarios venezolanos. Universitas Psychologica. Septiembre-Diciembre; V.7 (N³). Disponible http://www.scielo.unal.edu.co/scielo.php?script=sci_arttext\&pid=S1657926720080003000 $\underline{11 \& \operatorname{lng}=\mathrm{es} \& n r m=\mathrm{iso}}$

Kielhofner G. (2006). Fundamentos Conceptuales de la Terapia Ocupacional. $3^{\mathrm{a}}$ ed. Buenos Aires: Médica Panamericana.

Law M. Polatajko H. Baptiste S. (1997). Core concepts of occupational therapy. In Townsend, E. Enabling Occupation: An Occupational Therapy Perspective. Ottawa Ontario: CAOT Publications ACE.

Lazarus R. Folkman S. (1984). Stress, appraisal and coping. New York: Springer Verlag. 
Martínez M. (1996). Comportamiento Humano: nuevos métodos de investigación. 2ed. México: Trillas.

Orlandini A. (1999). El estrés, qué es y cómo evitarlo. México.

Pfeifer T. Kranz P. Scoggin A. (2008) Perceived Stress in Occupational Therapy Students. Occupational Therapy International. Septiembre; V.15 (Nº): [pp 221-231]

Polonio LB. Durante MP. Noya AB. (2001).Conceptos Fundamentales de Terapia Ocupacional. $1^{a}$ ed. Madrid: Médica Panamericana.

Rajab E. (2001) Effects of stress in dentists students. Journal of dent. Research. 69 (2). 120125

Román CC. Ortiz RF. Hernández RY. (2008). El estrés académico en estudiantes latinoamericanos de la carrera de Medicina. Cuba. Revista Iberoamericana de Educación. Julio; (N46). Disponible en: http://www.rieoei.org/deloslectores/2371Collazo.pdf

Sajjan K. Krupa J. (2005) Study of stress level in occupational therapy students during their academic curriculum. The Indian Journal Of Occupational Therapy. Vol. XXXVII ( $\left.{ }^{\circ} 1\right)$. Disponible en: http://medind.nic.in/iba/t05/i1/ibat05i1p11.pdf

Simó S. Urbanowski R. (2006) El Modelo Canadiense del Proceso del Desempeño Ocupacional. Revista Gallega de Terapia Ocupacional TOG. Febrero; (No3). Disponible en: http://www.revistatog.com/num3/pdfs/ExpertoI.pdf 